\title{
30 Doradus: The Low-Mass Stars
}

\author{
Hans Zinnecker \\ Astrophysikalisches Institut, An der Sternwarte 16, D-14482 Potsdam, \\ Germany
}

Bernhard Brandl

Cornell University, Department of Astronomy, Ithaca, NY 14853, USA

Wolfgang Brandner

JPL/IPAC, California Institute of Technology, 4800 Oak Grove Drive, Pasadena, CA 91109, USA

\section{Andrea Moneti}

ISO Science Operations Centre - VILSPA, Villafranca, 28080 Madrid, Spain

\section{Deidre Hunter}

Lowell Observatory, 1400 W. Mars Hill Road, Flagstaff, AZ 86001, USA

\begin{abstract}
.
We present a first analysis of our deep NICMOS/HST F160W images of the 30 Doradus cluster, aimed at detecting the low-mass stellar population $\left(\mathrm{M} \leq 2 \mathrm{M}_{\odot}\right)$. We find that the infrared luminosity function keeps rising towards the faint end and that there is no indication of a low-mass IMF cut-off down to at least $1.5 \mathrm{M}_{\odot}$. We also find a change of slope (steepening) in the luminosity function as a function of radial distance from the cluster center. The faintest stars we have detected have $\mathrm{H}=22.5 \mathrm{mag}$, corresponding to about $\mathrm{M}=0.4 \mathrm{M}_{\odot}$ at an age of $2 \mathrm{Myr}$.
\end{abstract}

\section{Introduction}

The low-mass stellar content in the 30 Doradus cluster (NGC 2070) is of major astrophysical interest for a number of reasons:

1. 30 Dor in the LMC is a massive, metal-poor young cluster, unlike any young cluster in our Galaxy. With hundreds of OB stars present, the question arises whether low-mass star formation is suppressed or not.

2. 30 Dor appears to be like a small young globular cluster. However, only if low-mass stars are present in sufficient numbers will the cluster remain bound and later develop into a globular cluster. 
3. 30 Dor may be the typical building block (unit cell) of violent star formation, now and in the high-redshift universe. Whether the IMF is low-mass deficient or not, makes a huge difference for the photometric and chemical evolution of young galaxies (e.g., Charlot et al. 1993; Zinnecker 1996; Larson 1998).

For earlier work on the IMF of this starburst cluster, we refer to Hunter et al. (1995), Brandl et al. (1996), and Massey \& Hunter (1998). Their work has resolved the high-mass and intermediate-mass stellar content of the cluster $(M \geq$ $3 \mathrm{M}_{\odot}$ ), with the result that the IMF closely follows a Salpeter mass distribution (slope $\Gamma=1.35$ ). The next step was to resolve the low-mass stellar population, using NICMOS on HST.

\section{Observations and Data Reductions}

The HST observations were carried out with the NIC2 camera (FOV $\left.\approx 20^{\prime \prime} \times 20^{\prime \prime}\right)$ in the $\mathrm{F} 160 \mathrm{~W}$ filter $\left(1.4-1.8 \mu \mathrm{m}\right.$, resolution $\left.0.15^{\prime \prime}\right)$ for maximum sensitivity and consisted of a $3 \times 3$ "core" mosaic centered on the cluster core, and two $3 \times 1$ "wings" extending away from the core region from two adjacent sides. In addition, the central core was also observed with NIC1 to provide improved spatial sampling of the very crowded center. Several sky observations were made in regions located $10-20^{\prime}$ north of the cluster core. All observations were obtained in MULTIACCUM mode with an integration time of up to 896 sec (the effective integration time can be much smaller as only the shortest MULTIACCUM readouts are used; see Zinnecker \& Moneti 1998 for more details and for a figure showing the layout of the observations superimposed on a NIR ground-based image). At each position four dithered images were obtained in order to remove bad pixels, cosmic rays, and the coronographic hole.

Individual frames were processed with the calnica (STSDAS) pipeline. Images were then cross-correlated in order to determine subpixel offsets, and stacked together using the DRIZZLE package. Remnant cosmic ray events, hot and bad pixels were masked out. Figure 1 shows a composite of the two wing mosaics.

Photometry was performed with DAOPHOT (Stetson 1987), and we present here preliminary results for wing2 (wing1, being much more crowded and containing many bright stars is much more difficult to analyze). The DAOPHOT analysis was performed in two runs: first the image shown in Figure 1 was analyzed, and second, the residual frame from the first run was boxcar smoothed and subtracted from the initial frame to produce a new frame free of nebulosity, and this image was analyzed. A preliminary photometric calibration was performed following the steps recommended in the STScI NICMOS photometry WWW pages. No color correction has been applied so far, and no attempt has been made to convert to a standard $\mathrm{H}$-band magnitude. With this preliminary calibration, the F160W magnitude of Mk34, a bright star near the core, is 12.0, i.e. $0.20 \mathrm{mag}$ larger than the $\mathrm{H}$ mag from Brandl et al. (1996). We consider this to be a good agreement at this stage, but that the final calibration could change by a few tenths of a magnitude.

Using this calibration we have extracted the F160W luminosity function (LF) for the wing2 field, shown in Figure 2. The main results are: 
1. the faintest stars detected using our two-step DAOPHOT process are 22.5 mag (corresponding to a pre-Main Sequence mass $\approx 0.4 \mathrm{M}_{\odot}$ for a cluster age of $2-4 \mathrm{Myr}$ and a distance modulus to the LMC of $18.5 \mathrm{mag}$ ).

2. the slope of the LF, i.e. $\log$ (number of stars) per unit H-magnitude, is 0.24 ; the slope appears to steepen slightly towards the outer edge of the field. Furthermore, there appears to be a feature in the LF at about $17.5 \mathrm{mag}$ which could correspond to the turn-on to the Main Sequence.

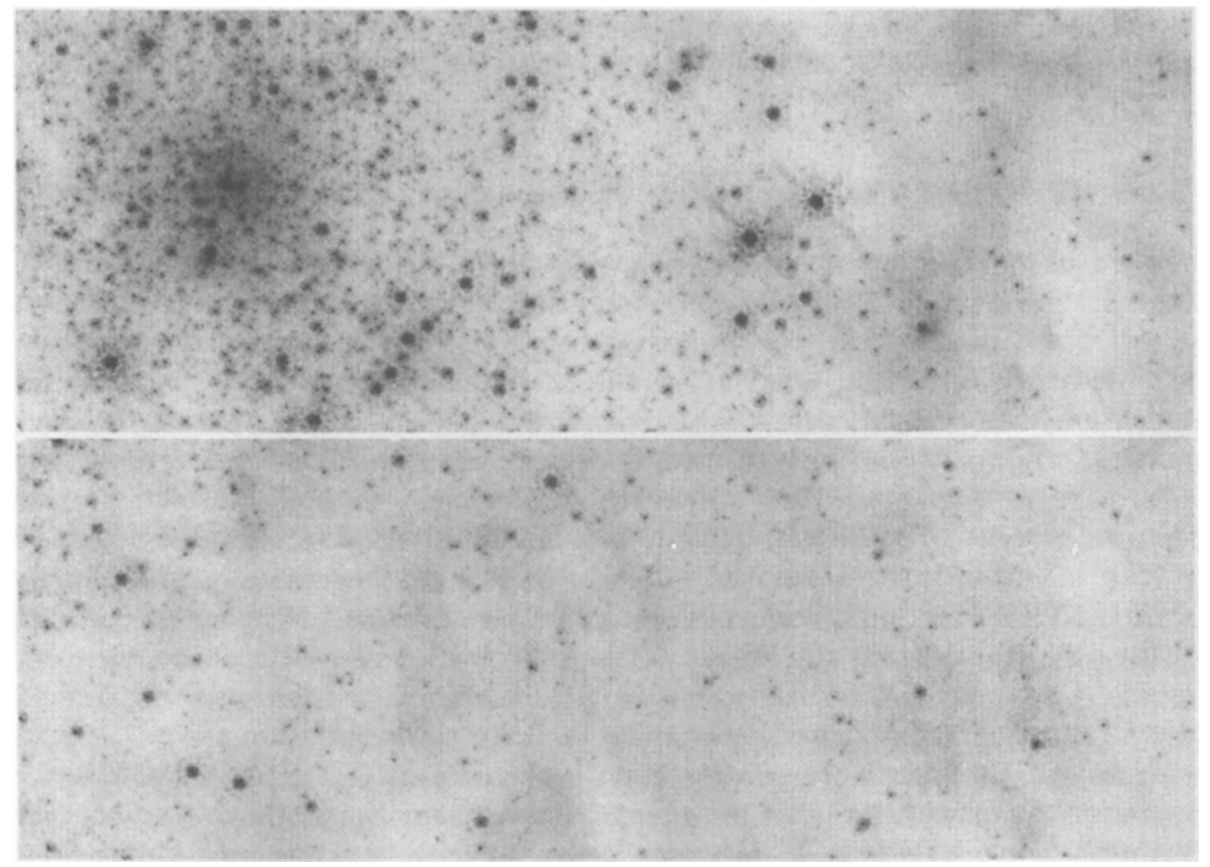

Figure 1. NICMOS/HST images (F160W filter) of the 30 Doradus cluster. Wing 1 (upper panel) contains the cluster core, wing 2 (lower panel) lies NW of the core. The horizontal width is $60^{\prime \prime}$ or $15 \mathrm{pc}$.

\section{Discussion}

We have presented the first infrared (H-band) luminosity function of the 30 Dor cluster, albeit not in the very cluster core but at radial distances $15-25 \mathrm{pc}$ where crowding is less severe. We have detected the first low-mass pre-MS stars below $1 \mathrm{M}_{\odot}(\mathrm{H} \approx 21)$. Although the luminosity function has so far not been corrected for incompleteness (and may therefore not exhibit the apparent turnover, but may still be rising at the faint end), it is likely to be largely complete to $\mathrm{H}=20$. Further ignoring a minor extinction correction and a very small correction for background contamination, we can convert the slope (s) of the 


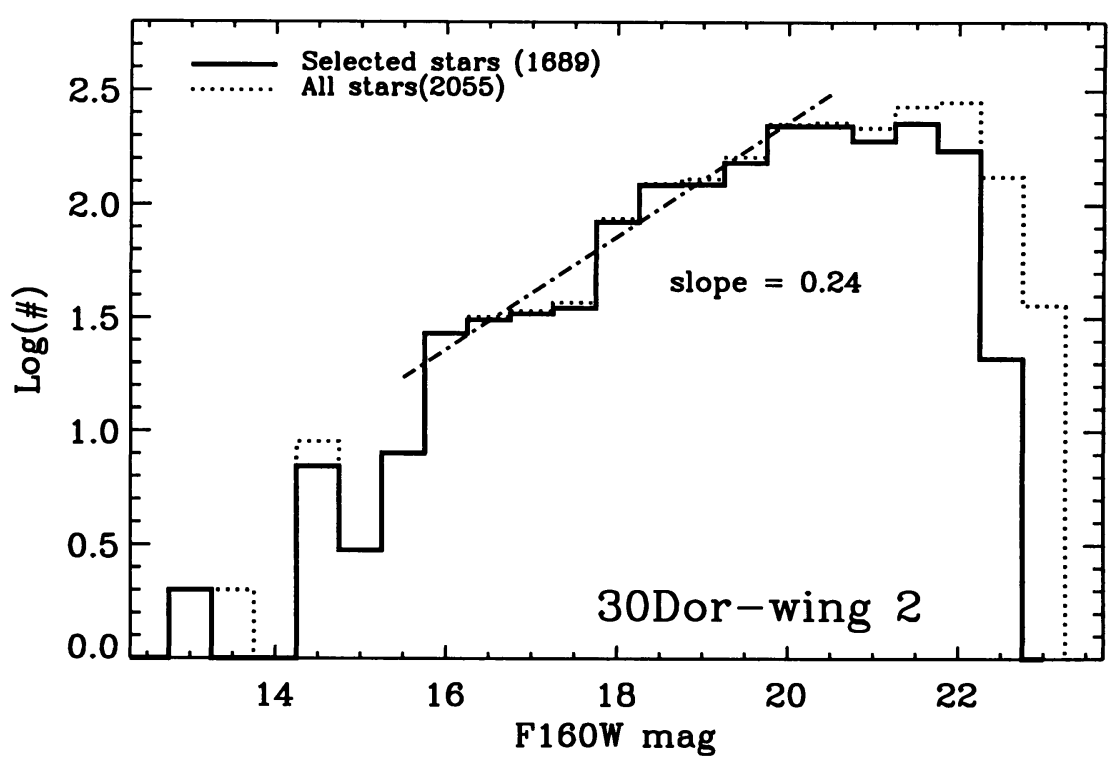

Figure 2. Infrared luminosity function (H-band, F160W) for Wing 2 in 30 Dor. Selected stars means that only stars with a magnitude error $\leq 0.2 \mathrm{mag}$ were considered (full line)

luminosity function between $\mathrm{H}=16$ and $\mathrm{H}=20$ (see Figure 2) to an IMF slope $(\Gamma)$, corresponding to a range of masses from 1.5 to about $10 \mathrm{M}_{\odot}$, using the time-dependent pre-MS mass-luminosity relation for the appropriate cluster age $\left(2 \mathrm{Myr}\right.$ ): $\mathrm{L}_{H} \sim \mathrm{M}^{1.4}$ (based on d'Antona \& Mazzitelli 1994 tracks and dwarf colors). We find $\Gamma=2.5 \times 1.4 \times s=0.84$ for $s=0.24$ (as derived from :w Figure 2 ). This IMF slope is very similar to that found in the young Galactic cluster NGC $3603(\Gamma=0.79)$ over a similar range of masses, based on adaptive optics observations (Eisenhauer et al. 1998). This is interesting, because of all young Galactic clusters NGC 3603 is the closest analog to the 30 Dor cluster in the LMC.

Acknowledgments. We thank our co-I's of the 30 Dor NICMOS project: M. McCaughrean, G.Meylan, R. Larson, M. Rosa, N. Walborn, and G. Weigelt. $\mathrm{BB}, \mathrm{WB}$, and DH acknowledge support by NASA through grant number GO7370.0 96A from STScI. HZ thanks the DFG for travel support which enabled him to participate in this enjoyable IAU Symposium in beautiful Victoria, Canada.

\section{References}

Brandl, B., Sams, B.J., Bertoldi, F., et al. 1996, ApJ, 466, 254

Charlot, S., Ferrari, F., Mathews, G.T., \& Silk, J. 1993, ApJ, 419, L57

d'Antona, F., \& Mazzitelli, I. 1994, ApJS, 90, 467

Eisenhauer, F., Quirrenbach, A., \& Zinnecker, H. 1998, ApJ, 498, 278 
Hunter, D.A., Shaya, E.J., Holtzman, J.A., et al. 1995, ApJ, 448, 179

Massey, P., \& Hunter, D.A. 1998, ApJ, 493, 180

Larson, R.B. 1998, MNRAS, 301, 569

Stetson, P.B. 1987, PASP, 99, 191

Zinnecker, H., \& Moneti, A. 1998, in NICMOS and the VLT, ESO Conf. \& Workshop Proc. No. 55, p. 136 (eds. W. Freudling \& R. Hook)

Zinnecker, H. 1996, in The interplay between massive star formation, the ISM, and galaxy evolution, eds. D. Kunth et al., p. 249

\section{Discussion}

Marc Kutner: As we are leaving the topic of star formation it is important to remember that stars are formed in molecular clouds. When we consider how some event may have caused or modified some star formation episode, we must consider the effect of the event on a parent cloud.

Zinnecker: Yes. We must also remember that molecular clouds often have a few dense cores from which star clusters or subgroups of stellar associations are forming, thus giving rise to hierarchical stellar clusterings.

Marc Kutner: How far down would the IMF have to cut off to bind the cluster (assuming a Salpeter IMF)?

Zinnecker: That depends on the mass of the original protocluster cloud and how much gas has been expelled from the cluster by the pressure of the HII regions and also massive stellar winds. The total stellar mass in the 30 Dor cluster in OBA stars down to $3 \mathrm{M}_{\odot}$ is about $2 \times 10^{4}$. For a Salpeter IMF the total stellar mass goes up by about a factor of 2 for a cut-off mass of $0.4 \mathrm{M}_{\odot}$. This could be sufficient to provide enough binding mass.

Nolan Walborn: To what mass does your completeness limit correspond?

Zinnecker: We think that we are about $80 \%$ complete to $\mathrm{M}=1.5 \mathrm{M}_{\odot}$.

Nolan Walborn: The dust, $\mathrm{H}_{2}$, and $\mathrm{CO}$ seen very near to $\mathrm{R} 136$ is likely in front of or behind it, at distances comparable to those of the material to the west and northeast, since it is a three-dimensional structure.

Kimmo Innanen: Can you measure the velocity dispersion from the spectra of the MASSIVE stars in 30 Doradus?

Zinnecker: This is difficult because of the line broadening in massive stars. Nonetheless, I understand that the experiment has been tried by Bosch, Terlevich, Melnick, and Selman; but I don't know the results. 